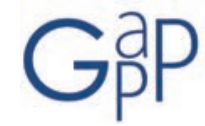

GESTIÓN Y ANÁLISIS DE POLÍTICAS PÚBLICAS, Nueva Época, nº 11 enero-junio 2014 ISSN: 1989-8991

DOI: http://dx.doi.org/10.24965/gapp.voi11.10178

\title{
Del pensamiento a la acción..., creando valor público. Herramientas de la gestión estratégica para la integración de las competencias en el Sistema Educativo
}

\author{
Paulino Martín Seco \\ Director del Instituto Nacional de las Cualificaciones (MECD) \\ paulino.martin@mecd.es \\ Sonia Martín Pérez \\ Profesora de la Escuela de Administración Pública de Castilla Y León (ECLAP) \\ sonia.martin.perez@hotmail.com
}

Recibido: 5 de febrero 2014

Aceptado: 26 de abril 2014

\begin{abstract}
Resumen
La evolución de los sistemas educativos en las últimas décadas, han llevado implícitos cambios en los espacios, en los medios, en los objetivos, pero sobre todo en los procesos. Esta evolución del proceso está ligada al desarrollo de la educación basada en competencias, que implica el desarrollo del aprendizaje por la vía de la experimentación, y que posibilita a las personas ser lo que puedan y quieran llegar a ser. Esta "novedosa" visión del aprendizaje, fue descrita por Confucio, quinientos años antes de nuestra era, "... me lo contaron y lo olvidé, lo vi y lo entendí, lo hice y lo aprendí...". Pero, evidentemente, en la actualidad las competencias necesarias para integrarse y desarrollarse en la sociedad, han cambiado, al igual que lo han hecho los objetivos, los medios y las necesidades.

En este artículo, proponemos la utilización de herramientas y modelos de gestión de la calidad para conseguir integrar la educación por competencias en todo el sistema educativo, desde la perspectiva de la gestión estratégica, alineando los objetivos de las políticas educativas actuales, de mejora de la calidad del sistema, de desarrollo económico y de cohesión social, con una metodología de gestión de dichas políticas, de forma que la mejora de las competencias básicas, profesionales e interpersonales sean el camino para alcanzar dichos objetivos, a través de políticas públicas que garanticen la eficacia, la eficiencia y la efectividad en la gestión de los recursos disponibles en la administración, para la generación de valor público en los ciudadanos y en la sociedad en su conjunto.
\end{abstract}

Palabras clave

Competencias, sistema educativo, estrategia, alineación estratégica, valor público, políticas públicas, administración pública.

\section{From the thought to the action..., creating public value. Strategic management tools for the integration of skills in the education system}

\begin{abstract}
The evolution of education systems in the last decades, changes have been implicit in places, in the media, on objectives, but especially in the process. This evolution in the process will be linked in the coming years to the development of competency-based education, which involves the development of learning via experimentation, and that enables people, be who can and will become. This new vision of learning was described by Confucius, five hundred years before our era, "... I was told and I forgot I saw it and understood it, I did and I learned ..." But obviously now the necessary competences to integrate and develop in society have changed, as did the goals, means and needs.

In this paper, we propose the use of tools and models of quality management for integrating competence education throughout the education system, from the perspective of strategic management, aligning the goals of current educational policies, to improve the system quality, economic development and social cohesion, with a management methodology such policies, so that the improvement of basic, professional and interpersonal competencies are the way to achieve these objectives through public policies that guarantee effectiveness, efficiency and effectiveness in the management of available resources in the administration, to generate public value for citizens and society as a whole.
\end{abstract}

Keywords

Competences, education system, strategy, strategic alignment, public value, public policy, public administration. 


\section{INTRODUCCIÓN}

Es notable la evolución que han sufrido los sistemas educativos desde que en 1948 la Declaración Universal de los Derechos Humanos ${ }^{1}$ enunciara la misión de la educación, y determinara que uno de los principales objetivos de la misma era el pleno desarrollo de la personalidad humana. En ese momento, se sentaron las bases de lo que hoy se reconoce como uno de los derechos fundamentales, avalado por todas las Constituciones de los países desarrollados, el derecho a la educación.

La educación básica y obligatoria, es recogida en nuestra Constitución², y en todas las leyes de educación, desde la Ley General de Educación (1970) hasta la actual Ley Orgánica para la Mejora de la Calidad Educativa (2014), pero los objetivos han variado para adaptarse a las necesidades sociales de cada momento.

Vivimos en un entorno en continuo cambio, donde la globalización y las tecnologías nos han llevado a la sociedad del conocimiento y de la información, y donde la crisis económica, el desempleo y la movilidad, hacen que los ciudadanos españoles tengamos que estar en permanente proceso de cualificación y recualificación, teniendo ahora más importancia que nunca el aprendizaje a lo largo de la vida.

Nuestro sistema educativo debe apoyar esta tarea por medio de sistemas flexibles, la variedad de itinerarios y la acreditación de los conocimientos formales, no formales e informales, que permitan la entrada al sistema en cualquier momento de la vida para adquirir las competencias necesarias y afrontar, desde una formación de calidad, los nuevos retos de la sociedad. Por ello, se hace necesario un cambio de modelo, tanto en el diseño, la planificación y el desarrollo de las políticas públicas como el proceso de enseñanza-aprendizaje, es preciso para conseguirlo la utilización de modelos y herramientas de gestión de la calidad aplicadas al sector educativo que permitan la integración de la educación por competencias en todos los niveles educativos.

Una vez expuesta la necesidad de dar un paso hacia la mejora de la calidad del sistema educativo se nos plantea la siguiente cuestión: ¿es posible adaptar esos modelos y herramientas a nuestro sistema educativo para llevar el aprendizaje basado en competencias a las políticas, los programas educativos y las aulas?

A lo largo de este artículo se irán dando las pautas para alcanzar dicho reto, pero primero en los dos siguientes apartados centramos brevemente los conceptos de competencia y estrategia, que posteriormente utilizaremos en el desarrollo de la solución del problema planteado.

\section{LAS COMPETENCIAS COMO GARANTÍA PARA EL ÉXITO DE LOS SISTEMAS DE EDUCACIÓN Y FORMACIÓN PROFESIONAL: ORIGEN Y FUTURO}

La introducción de las competencias no es algo nuevo, aunque sí de escasa implantación en determinados niveles educativos. Con la implantación de la $\operatorname{LOGSE}^{3}$ (1990) se introdujo el concepto de unidad de competencia como referente para la adquisición y evaluación de las capacidades terminales. Así, en la Educación Infantil, Primaria y Secundaria no ha tenido la misma relevancia como lo ha tenido en la Formación Profesional, a pesar de los intentos legislativos, como el de la LOCE4 (año), o la LOE5 (año), integrando las competencias básicas dentro de la definición y componentes del currículo de cada enseñanza como elementos vertebradores del mismo.

Para que el alumno adquiera estas competencias es necesario, en primer lugar, la determinación de un currículo de base o "core curriculum" por contra al tradicional método del currículo común o "commom curriculum", así como un cambio en la práctica docente desde la programación hasta la evaluación.

En un repaso rápido al concepto de competencias desde la perspectiva laboral, decir que éstas tienen su origen en los principios de la organización científica del trabajo (Taylor, 1912), cuando se plantea la necesidad de aumentar la producción maximizando el rendimiento de la mano de obra por medio de la división de las tareas, y la cualificación en partes concretas del proceso productivo. En los años 70, el Profesor David McClelland introdujo este concepto desde la perspectiva del desempeño en la dirección ejecutiva, como una herramienta para mejorar la productividad y el beneficio de las empresas basado no solo en el conocimiento, sino también en su experiencia y en sus habilidades (McClelland, 1975).

1 Artículo 26 de la Declaración Universal de los Derechos Humanos: "La educación tendrá por objeto el pleno desarrollo de la personalidad humana y el fortalecimiento del respeto a los derechos humanos y a las libertades fundamentales..."

2 Artículo 27.4 de la Constitución Española de 1978: "La enseñanza básica es obligatoria y gratuita".

3 LOGSE son las siglas de Ley de Ordenación General del Sistema Educativo.

$4 \quad$ LOCE son las siglas de Ley Orgánica de la Calidad Educativa.

5 LOE son las siglas de Ley Orgánica de Educación. 
En el ámbito educativo se trató la necesidad del trabajo por competencias en el proyecto DeSeCo ${ }^{6}$ de la OCDE (2005). El estudio plantea como método de trabajo la adquisición de un número pequeño de competencias que posibiliten la integración, la transferibilidad, y la aplicación de los conocimientos, de forma que la combinación conceptual, procedimental y actitudinal, en cualquier contexto y situación, permitan al individuo su desarrollo personal y social.

Tomando como referencia los estudios desarrollados por el "Partnership for $21^{\text {st }}$ Century Skills" en su modelo "Framework for $21^{\text {st }}$ Century Learning" (2009), las competencias se pueden clasificar en tres grandes grupos: básicas, profesionales e interpersonales. Para su consecución se requiere que el sistema educativo esté presente a lo largo de toda la vida del ciudadano, dado que no todas se puedan desarrollar en las etapas obligatorias y todas tienes una importancia capital en la vida de las personas de forma individual, para la cualificación y recualificación profesional, y colectiva, para su participación activa en la mejora de la sociedad, desde la perspectiva de la cohesión y del desarrollo económico.

En los siguientes apartados se describe cómo la introducción, diseño, desarrollo, implantación, seguimiento y evaluación de las competencias debe constituirse como una línea estratégica dentro de la educación y la formación, y por lo tanto, y para garantizar el logro de sus objetivos, debe alinearse estratégicamente con las distintas perspectivas del sistema educativo, partiendo de la definición de las políticas públicas, pasando por la implantación y ejecución de las mismas a través de la administración educativa y sus servicios, y finalizando con los resultados obtenidos, tanto a nivel individual en el alumno, como a nivel colectivo con su repercusión en la sociedad.

\section{ALINEACIÓN ESTRATÉGICA DE LAS POLÍTICAS PÚBLICAS: FUENTE DE CREACIÓN DE VALOR PÚBLICO}

Desde que Sun Tzu escribiera el tratado “El Arte de la Guerra”, 500 años antes de nuestra era, son muchos los autores que desde diferentes enfoques han determinado la importancia que tiene para las organizaciones la alineación entre los diferentes niveles de gestión, y estos a su vez con las necesidades y expectativas de sus clientes (Galvany, 2012).

Gran parte de los tratados tienen un marcado carácter militar, ya que fue en este ámbito donde se desarrolló inicialmente el concepto de estrategia como punto de partida para la realización del despliegue al campo operativo, aunque la mayor parte de ellos contienen además pensamientos filosóficos, culturales e incluso políticos y sociales, muy ligados a la educación de las personas.

Desde que en 1944 Johm Von Newman y Oskar Morgerstern, publicaran "Theory of games and economic behavior" (Teoría de juegos y comportamientos económicos), obra con la que se introdujo el concepto de estrategia, entendida ésta como los actos que desarrolla una empresa seleccionados de acuerdo a una situación concreta, y cuya idea básica es la mejora de la competencia, muchos has sido los autores que han profundizado en este concepto. Autores como Peter Drucker (La práctica del Management,1954), Alfred Chandler (Estrategia y Estructura, 1960), Michael Porter (Estrategia competitiva, 1980), Henry Mintzberg (El proceso estratégico, 1993) y R.S.Kaplan y D.P. Norton (El cuadro de mando integral, 1992 y 1996, Mapas estratégicos, 2004 y Alienación estratégica,2006), coinciden en sus teorías definiendo la estrategia de la organización como el corazón que hace que ésta se mueva hacia las metas fijadas a largo plazo, así como la importancia de la realización de un buen despliegue de la misma mediante la adopción de herramientas de gestión de la calidad y la asignación de recursos para alcanzar dichas metas, de forma eficaz, eficiente y efectiva, pero sin perder de vista la consecución de objetivos en el corto plazo.

El diccionario de la Real Academia de la Lengua Española (2001), define el término alinear como "poner en línea recta", y estrategia como "arte, traza para dirigir un asunto y proceso regulable, conjunto de las reglas que aseguran una decisión óptima en cada momento”. Uniendo ambos términos, alineación estratégica, será el arte de poner en línea recta el conjunto de decisiones uniendo el punto en el que se encuentra la organización con el punto al que quiere llegar, es decir, uniendo la misión con la visión.

Para trazar la línea o líneas estratégicas en la organización se tienen que determinar cuáles son los valores mediante los cuales serán capaces de crear valor, y que además ayuden a definir los objetivos estratégicos, tanto a largo plazo, como a medio y corto plazo, que formarán parte del mapa estratégico de la organización y de su cuadro de mando estratégico.

Si nos trasladamos al ámbito de la administración pública, la definición del concepto de "alineación estratégica" (Arieu 2007)7, implica alcanzar dicha alineación cuando los planes, programas y actuaciones que la admi-

6 Definición y Selección de Competencias.

7 Arieu define el concepto de "consistencia estratégica" y dice que ésta existe cuando las acciones de una organización son coherentes con las expectativas de la dirección, y estas a su vez lo son con el mercado y su entorno. 
nistración lleva a cabo están alineados con los compromisos del gobierno, y estos a su vez con las necesidades y expectativas de los ciudadanos.

Por otro lado, Joan Subirats (2008) define el concepto de "Políticas públicas" como "el conjunto de decisiones tomadas y acciones realizadas por una serie de actores, tanto públicos, como privados, orientadas hacia la resolución de un problema público claramente identificado", y define "Gestión pública" como, "el conjunto de decisiones y acciones realizadas por actores públicos, orientadas a la consecución de los resultados planteados en una política pública o en el desempeño de una actividad administrativa cotidiana". En conclusión, es precisa la definición de la estrategia para concretar objetivos a largo plazo, teniendo en cuenta las necesidades de los usuarios finales, y la asignación de los medios necesarios para la consecución de los mismos. La unión de estos conceptos es lo que representa el sistema político-administrativo.

En la Figura I, mostramos la representación gráfica de este sistema, en el vértice superior está el ciudadano, con una doble vertiente, la de contribuyente del sistema que decide en las urnas que partido político quiere que establezca las políticas públicas, y la de beneficiario, ya que recibe directamente los servicios que presta el mismo sistema a través de los distintos organismos de la administración pública.

De lo que los poderes públicos deciden, a lo que los directivos públicos despliegan, y de lo que los funcionarios públicos ejecutan, a lo que finalmente los ciudadanos reciben, existe en algunos casos unos desajustes insalvables. Por tanto, es imprescindible la alineación estratégica ${ }^{8}$ de las políticas públicas con la dirección y administración pública, reduciendo al máximo esos desajustes y siendo una fuente de creación de valor público9.

\section{FIGURA I. TRIÁNGULO DE DESAJUSTES DEL SISTEMA CIUDADANO-POLÍTICO-ADMINISTRATIVO}

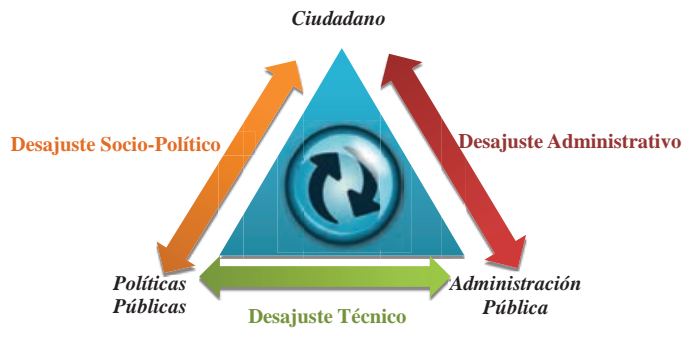

Fuente: elaboracion propia

Una vez definidos el concepto de competencia por un lado, y el de estrategia por otro, pasamos a definir, en base a las herramientas de esta última, un modelo que facilite la alineación de las políticas educativas en referencia al desarrollo del currículo competencial en el sistema educativo.

\section{MOdELO de alineación estratécica para el sistema educativo desde el enfoque COMPETENCIAL}

A continuación se resumen los aspectos más relevantes del modelo planteado, y su aproximación práctica, utilizando para su definición los elementos estratégicos más extendidos (Ronda Pupo, Guillermo A. y Marcané Laserra, José Ángel, 2004).

\section{Principios y fundamentos}

Inspirado en los principales modelos de gestión y evaluación como las normas ISO 9000:2008, el Modelo LEAN $^{10}$, el Modelo EFQM ${ }^{11}$ y el Modelo de Dirección y Gestión Pública ${ }^{12}$ y aplicando los principios del Buen Gobierno y la Buena Administración ${ }^{13}$, obtenemos los principios básicos en los que se fundamenta el modelo y que se recogen en la Tabla I.

8 La alineación estratégica se apoya en los cinco principios activos de sus teorías, que se centran en la movilización del cambio a través del liderazgo, la traducción de la estrategia en términos operativos, la alineación de la organización con la estrategia, y en hacer de la estrategia un trabajo continuo y de todos. (Kaplan y Norton, 2006).

9 Moore, Mark H (1998), dice que el valor público “consiste en generar el máximo valor posible para la población a partir de los recursos financieros, humanos, físicos y tecnológicos existentes en la institución, basándose en la imaginación gerencial, a fin de cumplir con los propósitos establecidos en los mandatos y con la máxima eficacia y eficiencia".

10 Lean Thinking, es un modelo de gestión desarrollado por Toyota que implica a todos los empleados a crear en todos los procesos valor para los clientes.

11 Modelo EFQM (European Foundation for Quality Management) de calidad y excelencia es una vía para la autoevaluación y la determinación de los procesos de mejora continua en entornos empresariales tanto privados como públicos.

12 Premio de Investigación sobre Modernización de la Administración de la Comunidad de Castilla y León. Año 2006

13 Aspectos esenciales de la Ley 9 Ley 19/2013, de 9 de diciembre de Transparencia, Acceso a la Información Pública y Buen Gobierno GESTIÓN Y ANÁLISIS DE POLÍTICAS PÚBLICAS, Nueva Época, nº 11 enero-junio 2014 ISSN: 1989-8991 - DOI: 10.24965/gapp.v0i11.10178 
Sobre la premisa de la creación de Valor Público a través de la excelencia pública, en el marco de una democracia de calidad en equidad, para alcanzar la cohesión y el bienestar social de forma sostenida, establecemos los términos de la ecuación de generación de Valor Público que queremos maximizar por medio de la implantación del modelo:

[Open Governance + Good Governance $=$ Public Value $]$

\section{TABLA I. PRINCIPIOS Y ACCIONES DE LA FUNCIÓN DE GENERACIÓN DE VALOR PÚBLICO}

TABLA I. Principios y acciones de la función de generación de valor público

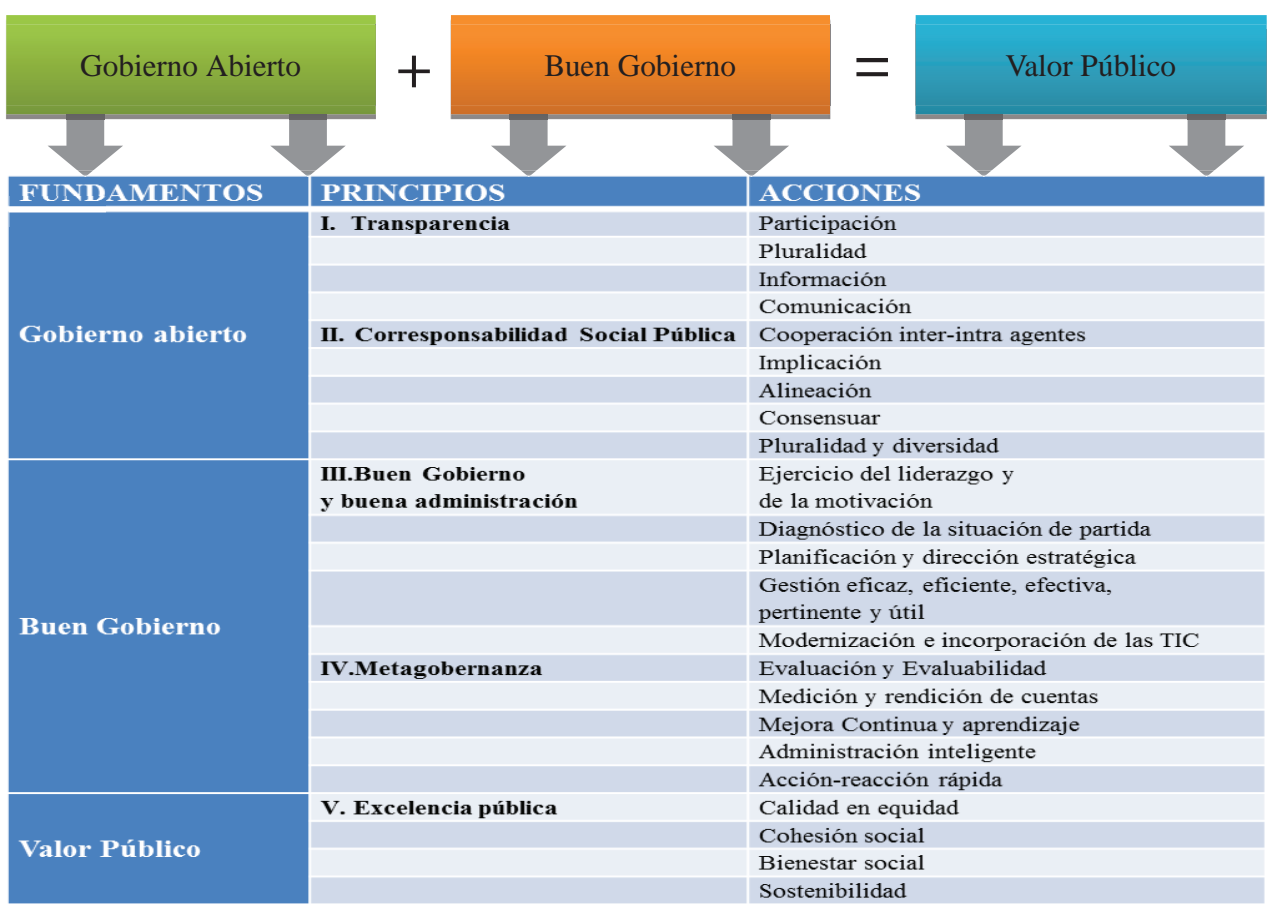

Fuente: elaboracion propia

\section{Objeto}

El objetivo fundamental del modelo, cuyo diagrama general de proceso se detalla en la Figura II, es maximizar la función de obtención de valor público aumentando el impacto de la ejecución de las políticas educativas a través de la aplicación de métodos y herramientas innovadoras del campo de la ingeniería y la administración de empresas, que permitan pasar de la estrategia establecida en la agenda gubernamental al plano operativo, es decir, de la toma de decisiones a los planes de acción, siguiendo una "línea estratégica" que cumpla las condiciones de mayor rendimiento, ex ante y ex post ${ }^{14}$, marcadas por la Unión Europea. Y, realizando un seguimiento y control del desempeño estratégico que permita reorientar los esfuerzos hacia los resultados esperados mediante la monitorización de los procesos, y su reajuste con el escenario estratégico diseñado, finalizando con la evaluación de las políticas y de su impacto de forma integral.

La lectura de este diagrama se podría resumir en que: tras la detección en el ámbito político y estratégico de una necesidad de los grupos de interés (cajas 1 a 8) y definición de cuál sería la mejor solución a la misma (cajas 9 y 10), se ha de definir los programas y actuaciones que permitan cubrir la citada necesidad (cajas 11 a 13). Para el desarrollo de estos hay que dotar de los recursos necesarios (cajas 14 a 19) y de los procesos operativos concretos (caja 18) y finalmente establecer los mecanismos de seguimiento y evaluación que permitan determinar su progreso y la consecución de los objetivos previstos (cajas 20 a 24). Las flechas grises indican la secuencia de actividades y las rojas representan los tipos de indicadores que permiten el seguimiento y evaluación.

14 Política de Cohesión 2014-2020. Inversión en el crecimiento y el empleo 
FIGURA II. MODELO DE ALINEACIÓN ESTRATÉGICA PARA LA CREACIÓN DE VALOR PÚBLICO. CADENA IPO (INPUT-PROCES-OUTPUT/ ENTRADAPROCESO/RESULTADO) DE APLICACIÓN



Fuente: elaboracion propia

Arquitectura y elementos del modelo: El Cubo Estratégico

Una vez descrita la finalidad y los fundamentos del modelo en los apartados anteriores, el siguiente paso será presentar su estructura y los elementos que lo componen, para explicar cómo se integrarían en nuestro sistema educativo.

El primero de los elementos lo hemos denominado "cubo estratégico" y su finalidad es concretar los aspectos fundamentales de la estrategia a configurar en materia de integración de las competencias en el sistema educativo, y que son definir la misión, la visión y valores de la misma y las herramientas de la calidad a utilizar.

El cubo estratégico (Figura III) de forma gráfica, representa un sistema de coordenadas en el que situamos en el eje de ordenadas las perspectivas del modelo, en el eje de abscisas los ejes estratégicos, y en el eje perpendicular los pasos a seguir para la generación de valor público propuestos en el modelo de alineación estratégica. 




Fuente: elaboracion propia

Las diferentes secciones del cubo representado recogen el despliegue de la estrategia desde su fase inicial, punto de partida o MISIÓN, hasta llegar pasando por la acción al punto final o VISIÓN a través de las líneas estratégicas, con las diferentes herramientas utilizadas en cada caso.

En el presente artículo nos centraremos en la definición de los elementos generales y de las primeras herramientas, es decir del mapa y del cuadro de mando estratégico.

\section{Misión, Visión y Valores}

Desde el punto de vista teórico ${ }^{15}$ se definen la misión y la visión de una organización de la siguiente forma:

- Misión: Es la razón de ser de una organización o sistema, debiendo recoger las funciones, servicios o actividades de la organización e incluir los grupos de interés de la misma.

- Visión: Son los objetivos globales que suponen un reto a la organización para un período de tiempo determinado. A partir de la misma se desarrollan la planificación estratégica y se focalizan los esfuerzos definiendo a donde queremos llegar.

- Valores: recogen el conjunto de principios y normas que forman parte cultura de la organización en forma de filosofía institucional, que ayudan a fijar un marco corporativo que guía las actuaciones de una organización

Desde estas definiciones y teniendo en cuenta que por un lado el Consejo de Ministros de la UE, en su reunión del día 12 de mayo de $2009^{16}$, adoptó el nuevo Marco para la cooperación europea en educación y formación, Estrategia Europea 2020, que se centra en alcanzar un crecimiento inteligente, sostenible e integrador, mediante el desarrollo de los cuatro objetivos estratégicos definidos para el ámbito educativo por la Comisión Europea acordes con los trabajos de la OCDE y de la UNESCO:

- Hacer realidad el aprendizaje permanente y la movilidad de los educandos.

- Mejorar la calidad y la eficacia de la educación y la formación.

- Promover la equidad, la cohesión social y la ciudadanía activa.

- Incrementar la creatividad y la innovación, incluido el espíritu empresarial, en todos los niveles de la educación y la formación.

La misión del sistema educativo actual se puede enunciar de la siguiente forma:

15 Martín Pérez, S. y Martín Seco, P. (2014), La excelencia operativa en la Administración Pública Creando Valor Público: Guía para la implantación de la gestión basada en procesos en la Administración Pública. Madrid: INAP

16 Publicados en el Diario Oficial de la Unión Europea 2009/C 119/02

17 Respetando plenamente la responsabilidad de los Estados miembros en relación con sus sistemas de enseñanza. GESTIÓN Y ANÁLISIS DE POLÍTICAS PÚBLICAS, Nueva Época, nº 11 enero-junio 2014 ISSN: 1989-8991 - DOI: 10.24965/gapp.v0i11.10178 
Ser capaz de conseguir el máximo desarrollo competencial de las personas, en todas sus dimensiones; científica, profesional y social, teniendo en cuenta la diversidad del alumnado, en cuanto a sus circunstancias personales y sociales, así como a sus necesidades y expectativas, facilitando su empleabilidad y participación activa en una sociedad democrática, de forma que contribuya a aumentar la productividad y competitividad del país, alcanzando de esta forma la sostenibilidad económica y la cohesión social.

Y, por otro lado y como punto hasta el que queremos que evolucione el Sistema Educativo español a través de las estrategias planteadas, tomaremos los objetivos marcados por la LOMCE:

- Situar a los alumnos como centro y razón de ser se la educación.

- Convertir la educación en el principal instrumento de movilidad social.

- Favorecer una educación de calidad como soporte de la igualdad, la equidad y la justicia.

- Facilitar el desarrollo personal y profesional, la integración social y la formación integral.

- Adecuar el Sistema Educativo a las nuevas demandas de aprendizaje por competencias, el aprender haciendo y el aprendizaje a lo largo de la vida.

- Luchar contra la exclusión que provoca las altas tasas de abandono escolar temprano y los bajos niveles de calidad.

- Situar la educación como el motor que promueve el bienestar de un país.

La visión del sistema educativo sería la siguiente:

El Sistema Educativo tiene como vocación ser el principal instrumento de la cohesión social, favoreciendo una educación de calidad como soporte de la equidad, luchando a favor de la inclusión social activamente, y sirviendo de motor para promover el bienestar social del país, así como impulsando la cultura del esfuerzo, el trabajo y la excelencia entre sus ciudadanos, a través de la adquisición de las competencias básicas y profesionales, y la mejora de sus competencias interpersonales a lo largo de toda su vida.

Por tanto, los Valores específicos para el sistema educativo ${ }^{18}$, además de los recogidos en la Tabla I son:

- Autonomía de los centros

- $\quad$ Rendición de cuentas

- Atención a la diversidad

\section{Perspectivas}

Kaplan y Norton ${ }^{19}$ plantean en su teoría para la creación de Mapas Estratégicos (ME) y Cuadros de Mando Integral (CMI), una estructura de perspectivas que responde a la organización de los resultados que genera la actividad de una organización empresarial en cuatro aspectos de interés estratégico para la misma.

Adaptando este modelo a la administración pública hemos determinado nuevas perspectivas que se ajustan mejor a la misión y visión de las organizaciones públicas y hemos reordenado la posición de las mismas poniendo el foco de atención en el ciudadano.

Las cuatro perspectivas utilizadas en el Modelo se dividen en resultados e inductoras, y son:

\begin{tabular}{|c|c|}
\hline Resultados & Inductoras \\
\hline $\begin{array}{c}\text { Sociedad } \\
\text { Ciudadano/Alumnado }\end{array}$ & Administración Educativa \\
& Políticas Educativas \\
\hline
\end{tabular}

Estas cuatro perspectivas se pueden desagregar a su vez en diferentes segmentos que ayudarán a realizar el diseño del Mapa de forma más ordenada, así como a determinar las interrelaciones o relaciones causa-efecto entre sus nodos, tal y como se recoge en la Tabla II.

18 Fundamentados en la Ley Orgánica 8/2013, de 9 de diciembre, para la mejora de la calidad educativa.

19 Perspectivas del CMI y del ME: Perspectiva financiera, perspectiva del cliente, perspectiva de los procesos internos y perspectiva del aprendizaje y del crecimiento. R.S. Kaplan y D.P. Norton (2001 y 2007)

GESTIÓN Y ANÁLISIS DE POLÍTICAS PÚBLICAS, Nueva Época, nº 11 enero-junio 2014 ISSN: 1989-8991 - DOI: 10.24965/gapp.v0i11.10178 
TABLA II. DESAGREGACIÓN DE LAS PERSPECTIVAS DEL MAPA ESTRATÉGICO

\section{PERSPECTIVAS DEL MAPA ESTRATÉGICO}

\begin{tabular}{|c|c|c|}
\hline กิ & Sociedad & $\begin{array}{l}\text { Entorno laboral y empresarial } \\
\text { Agentes sociales } \\
\text { Asociaciones y Colectivos } \\
\text { Ciudadanos }\end{array}$ \\
\hline 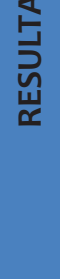 & Ciudadano/Alumnado & $\begin{array}{l}\text { Educación a lo largo de la Vida } \\
\text { Educación para Adultos } \\
\text { Educación Superior } \\
\text { Educación Secundaria postobligatoria } \\
\text { Educación Básica }\end{array}$ \\
\hline $\begin{array}{l}\text { u } \\
\text { 品 } \\
\text { o }\end{array}$ & Administración Educativa & $\begin{array}{l}\text { Centros y Servicios Educativos } \\
\text { CCAA-Consejería de Educación, Direcciones Provinciales de Educación } \\
\text { Ministerio de Educación }\end{array}$ \\
\hline 号 & Políticas Educativas & $\begin{array}{l}\text { Gobierno de las CCAA } \\
\text { Gobierno de España } \\
\text { Unión Europea }\end{array}$ \\
\hline
\end{tabular}

Fuente: elaboracion propia

\section{Ejes estratégicos}

Los ejes serán los elementos vertebradores del modelo. Éstos nos permitirán atravesar las perspectivas fijadas particularizando su contenido para cada organización de forma ordenada e interrelacionada. Para su elección podemos tener en cuenta las directrices, los agentes, los procesos, las funciones o cualquier otro elemento en el que se estructure la organización, sistema o materia que estemos trabajando.

Los ejes que vamos a utilizar para vertebrar el caso que nos ocupa los obtenemos de la agrupación de los grandes bloques en los que se estructura el sistema educativo y su ordenación, la Educación Básica, la Formación Profesional y la Formación a lo largo de la vida. Estos a su vez están estrechamente ligados a los tres tipos de competencias que forman parte del desarrollo integral del ciudadano, nos referimos a las competencias básicas, las competencias profesionales y las competencias interpersonales.

\section{Línea estratégica}

Son la concreción de la estrategia y definen la relación entre las distintas perspectivas. Nos ayudan a unir los objetivos estratégicos definidos en las diferentes perspectivas, desde el punto de partida o misión al punto objetivo o visión, dando sentido a las propuestas de acción estratégicas de la organización, y representando las relaciones causales como herramienta de comunicación y aprendizaje.

Las tres líneas estratégicas serán:

- $\quad$ EDUCA: en referencia a la educación básica y a las competencias básicas.

- $\quad$ FORMA: en referencia a la formación profesional y a las competencias profesionales.

- INTEGRA: en referencia a la formación a lo largo de la vida y a las competencias interpersonales.

El dinamismo que queremos conseguir para hacer que el sistema educativo evolucione de su situación actual a la situación objetivo lo trabajamos a través del estudio de las relaciones que se producen entre las diferentes estrategias (denominadas relaciones causa-efecto), los objetivos estratégicos y la interrelación entre los indicadores de los diferentes procesos ${ }^{20}$, tal y como se representa en la Figura IV.

20 Los indicadores pueden ser inductores (asociados a la estrategia), y se agrupan en el cuadro de mando estratégico o de resultado GESTIÓN Y ANÁLISIS DE POLÍTICAS PÚBLICAS, Nueva Época, nº 11 enero-junio 2014 ISSN: 1989-8991 - DOI: 10.24965/gapp.v0i11.10178 


\section{FIGURA IV. INTEGRACIÓN DEL MODELO Y LAS PERSPECTIVAS CON EL MAPA DE PROCESOS GENÉRICOS DEL SISTEMA EDUCATIVO}
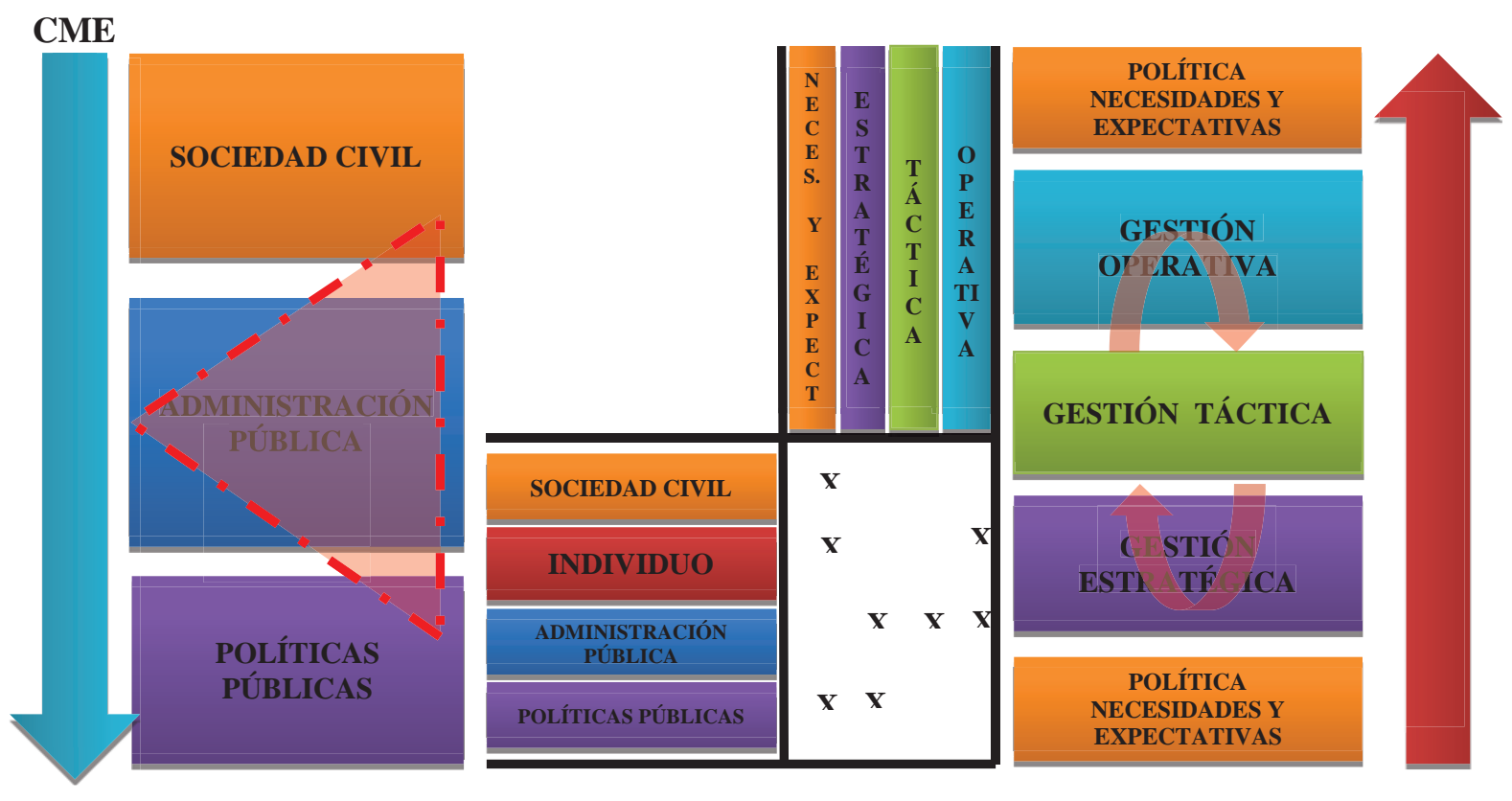

CMI

Fuente: elaboracion propia

\section{Mapa Estratégico (ME) del Sistema Educativo desde el enfoque competencial}

Es la representación gráfica de las estrategias globales de una organización y sus interrelaciones. Cada una de las estrategias, nodos del grafo, se situará en el mapa en una de las cuatro perspectivas y corresponderá a uno de los ejes definidos. Y la unión entre las diferentes estrategias, nodos, que representan las relaciones causales, son las líneas estratégicas.

La Figura VI representa el primer hito en la trayectoria de generación de valor que se alcanza con la definición del Mapa Estratégico y cuya lectura se concreta en:

Queremos un Sistema Educativo de calidad, en equidad, en pro del desarrollo económico sostenido y la cohesión social...(SOCIEDAD)

... y para conseguirlo aumentaremos las competencias del alumnado mejorando así sus resultados académicos y promoviendo el desarrollo integral de los ciudadanos a través de su cualificación y recualificación profesional... (ALUMNADO)

... apoyados en un Sistema de Gestión Excelente de los centros y servicios educativos,

que centre sus esfuerzos en el desarrollo competencial del alumnado, en la preparación del profesorado y en la adaptación a los nuevos procesos y medios...(ADMINISTRACIÓN EDUCATIVA)

... partiendo del impulso de políticas educativas encaminadas al diseño, planificación y desarrollo competencial de todas las enseñanzas, la potenciación de aquellas que posibilitan la empleabilidad y atendiendo a la diversidad de necesidades, capacidades, intereses y motivaciones de los ciudadanos. (POLÍTICAS PÚBLICAS)

(asociados a los procesos) que se agrupan en el cuadro de mando integral. 


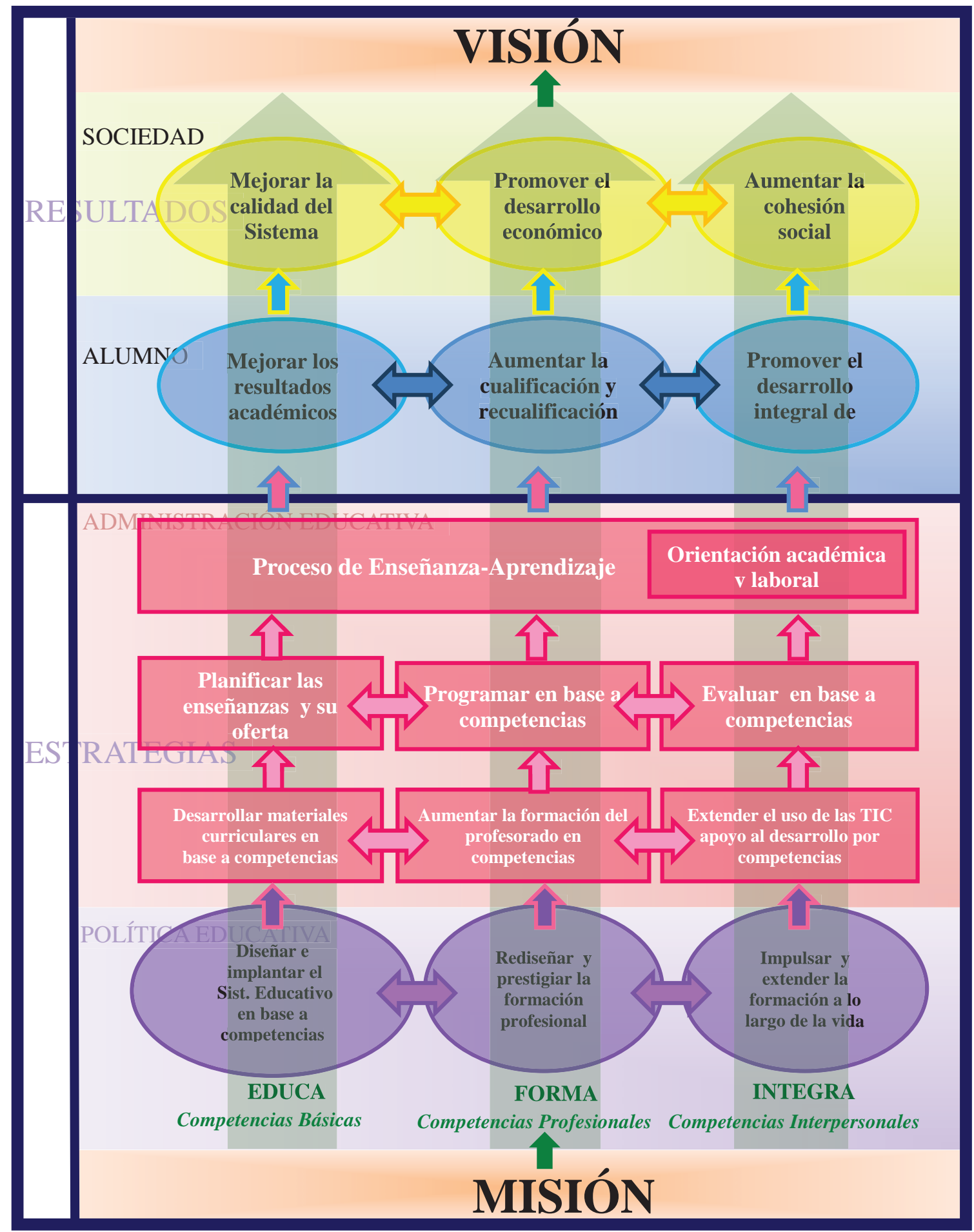

Fuente: elaboracion propia

\section{Cuadro de Mando Estratégico (CME) del Sistema Educativo desde el enfoque competencial}

La concreción de las estrategias en objetivos específicos, y la asociación a éstos de indicadores inductores, distribuidos en las diferentes perspectivas e integrados en las líneas estratégicas, conforman el CME

Conocidos los objetivos estratégicos y los indicadores asociados a los mismos, hemos de saber medir en referencia a ellos, y actualizar nuestro CME y nuestros objetivos a mejorar, para ello hemos de asignar a cada indicador una serie de parámetros como son el origen de la información, el punto de partida de dichos parámetros, el objetivo que deseamos alcanzar, y quienes son los distintos responsables de recopilar, gestionar y revisar los citados 
parámetros. Todos los indicadores se recogen en una tabla, en la que aparecerá, el indicador y su designación, el objetivo al que pertenece, y la perspectiva correspondiente, para situarlo en el contexto global.

El valor de un objetivo se hallará en relación a los indicadores que tiene asociados, en función de métodos matemáticos (la media de ellos) o cuantitativos (indicadores sintéticos, que implican la interrelación entre diversos indicadores del mismo objetivo o de otros), de la experiencia (resultados de otros años) o de los referentes normativos a cumplir en cada caso (ratios, horas docencia).

En la citada tabla también se incluyen los factores de medición para el control de los indicadores, como son el valor real o actual ( Vrr) y el valor objetivo (Vo). Para que el cuadro sea más gráfico, se ha adoptado un código de colores para determinar el estado del objetivo en conjunto y de los indicadores de forma individual, siendo por ejemplo (Tabla III) el verde, un valor óptimo, el amarillo un valor aceptable y el rojo un valor inapropiado, obteniendo, de forma visual, la configuración total del mapa como punto fuertes, áreas de mejora y punto débiles, respectivamente. $Y$, poder utilizar esta información para acometer las medidas oportunas y conseguir su corrección. Las interrelaciones anteriormente citadas también nos sirven para determinar qué aspectos tienen más incidencia sobre los objetivos y poder priorizar o potenciar estos, actuando con mayor eficacia y eficiencia.

EI CME, también se puede dotar de un simulador que permita la introducción de valores de simulación (Vs) y que nos aporte el valor del objetivo simulado (Vos), pudiendo así realizar pruebas de cómo la variación de determinados indicadores de forma conjunta aporta valor al objetivo y optimizar así la asignación de recursos, convirtiéndose en una valiosa herramienta para la toma de decisiones.

TABLA III. EJEMPLO DE APLICACIÓN DEL CUADRO DE MANDO ESTRATÉGICO

\begin{tabular}{|c|c|c|c|c|c|}
\hline \multirow{3}{*}{ 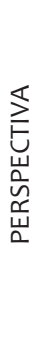 } & OBJETIVO ESTRATÉGICO & ValorObjetivo & Valor Real & Valor Simulación & $\begin{array}{c}\text { Valor Objetivo } \\
\text { Simulado }\end{array}$ \\
\hline & INDICADOR-1 & $\begin{array}{c}\text { Vo } \\
\text { INDICADOR-1 }\end{array}$ & $\begin{array}{c}\mathrm{Vr} \\
\text { INDICADOR-1 }\end{array}$ & $\begin{array}{c}\text { Vs } \\
\text { INDICADOR-1 }\end{array}$ & $\begin{array}{c}\text { Vos } \\
\text { INDICADOR-1 }\end{array}$ \\
\hline & INDICADOR-n & $\begin{array}{c}\text { Vo } \\
\text { INDICADOR-n }\end{array}$ & $\begin{array}{c}\mathrm{Vr} \\
\text { INDICADOR-n }\end{array}$ & $\begin{array}{c}\text { Vs } \\
\text { INDICADOR-n }\end{array}$ & $\begin{array}{c}\text { Vos } \\
\text { INDICADOR-n }\end{array}$ \\
\hline
\end{tabular}

A partir del Mapa Estratégico descrito en la Figura VI, y de los indicadores más relevantes de educación establecidos por la OCDE, por la Comisión Europea ${ }^{21}$, por el Ministerio de Educación, Cultura y Deporte a través del Mapa de Indicadores del INEE (Instituto Nacional de Evaluación Educativa) y otros (PISA, OCDE, CIS, EPA...), hemos diseñado el Cuadro de Mando Estratégico que será la herramienta que utilicemos para desplegar e implementar la estrategia en la organización y que recogen en la Tabla IV.A, B y C.

21 Consejo de Ministros 2007, "Core Indicators", conjunto de 16 indicadores clave. 
TABLA IV.A. CUADRO dE MANDO ESTRATÉGICO DEL SISTEMA EDUCATIVO EN BASE A LA LíNEA ESTRATÉGICA DE LAS COMPETENCIAS. RESULTADOS

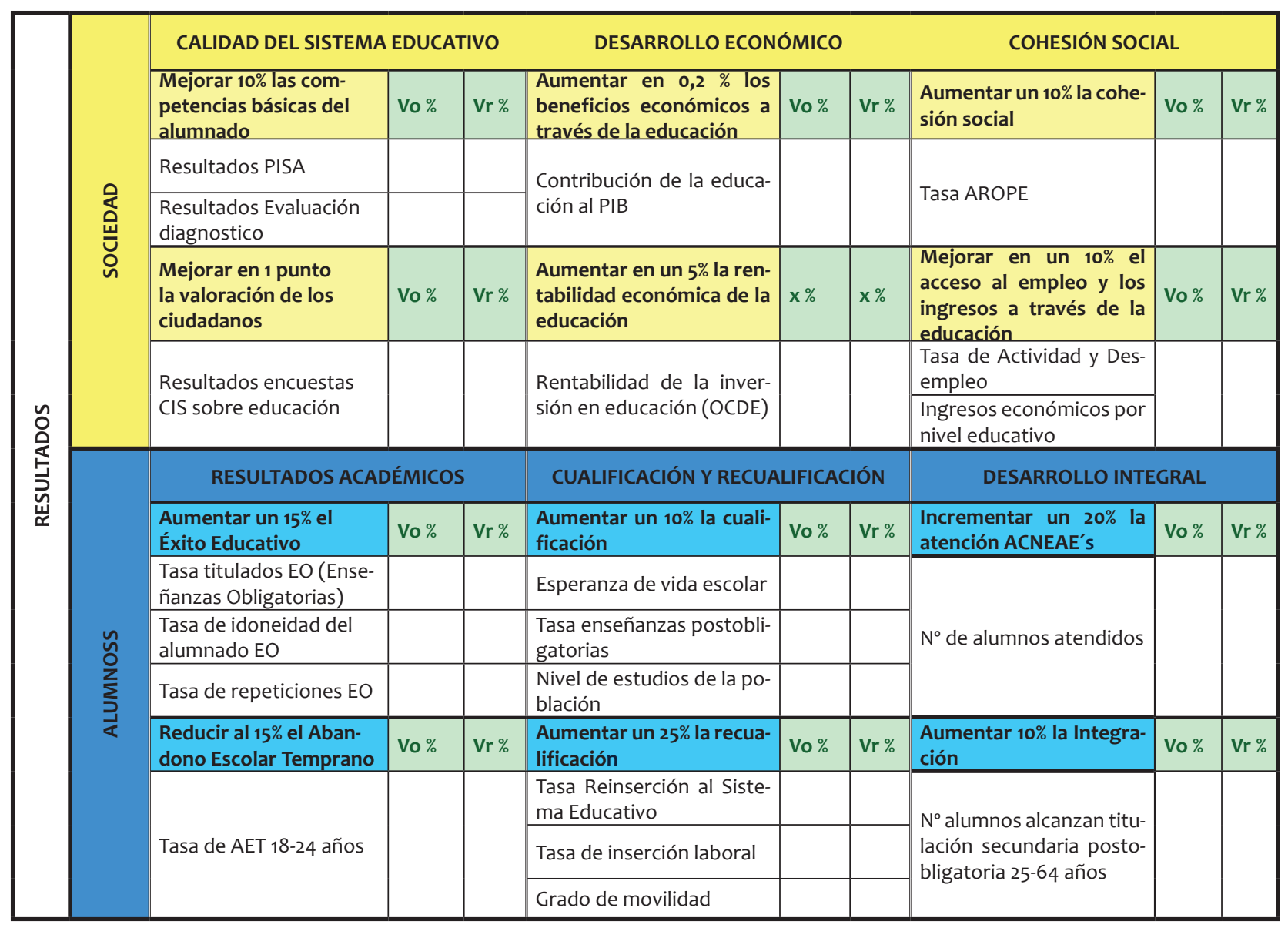


TABLA IV.B CUADRO DE MANDO ESTRATÉGICO DEL SISTEMA EDUCATIVO EN BASE A LA LÍNEA ESTRATÉGICA DE LAS COMPETENCIAS. ESTRATEGIAS. ADMINISTRACIÓN EDUCATIVA

\begin{tabular}{|c|c|c|c|c|c|c|c|c|c|c|}
\hline \multirow{10}{*}{  } & & \multicolumn{3}{|c|}{ PROCESO DE ENSEÑANZA APRENDIZAJE } & \multicolumn{3}{|c|}{ PROFESORADO } & \multicolumn{3}{|l|}{ RECURSOS } \\
\hline & \multirow{9}{*}{ 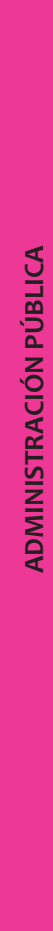 } & $\begin{array}{l}\text { Evaluar el aprendizaje } \\
\text { en base a competen- } \\
\text { cias }\end{array}$ & Vo \% & $\operatorname{Vr} \%$ & $\begin{array}{l}\text { Crear comunidades de } \\
\text { aprendizaje en entor- } \\
\text { no web }\end{array}$ & Vo \% & $\operatorname{Vr} \%$ & $\begin{array}{l}\text { Establecer convenios de } \\
\text { colaboración con empresas } \\
\text { editoriales }\end{array}$ & Vo $\%$ & Vr \% \\
\hline & & $\begin{array}{l}\mathrm{N}^{\circ} \text { de pruebas dise- } \\
\text { ñadas }\end{array}$ & & & $\begin{array}{l}\mathrm{N}^{\circ} \text { de profesionales } \\
\text { que participan }\end{array}$ & & & \multirow{2}{*}{$\mathrm{N}^{\circ}$ de convenios establecidos } & & \\
\hline & & $\begin{array}{l}\text { \% de pruebas aplica- } \\
\text { das/diseñadas }\end{array}$ & & & $\begin{array}{l}\mathrm{N}^{\circ} \text { de iniciativas pues- } \\
\text { tas en marcha }\end{array}$ & & & & & \\
\hline & & $\begin{array}{l}\text { Elaborar y desarrollar } \\
\text { de forma coordinada } \\
\text { la programación de } \\
\text { aula }\end{array}$ & Vo \% & $\operatorname{Vr} \%$ & $\begin{array}{l}\text { Desarrollar planes de } \\
\text { formación individual } \\
\text { y de centro en compe- } \\
\text { tencias y TIC's }\end{array}$ & Vo \% & $\operatorname{Vr} \%$ & $\begin{array}{l}\text { Desarrollar materiales curri- } \\
\text { culares } 2.0\end{array}$ & Vo $\%$ & Vr $\%$ \\
\hline & & $\begin{array}{l}\mathrm{N}^{\circ} \text { de programaciones } \\
\text { desarrolladas }\end{array}$ & & & $\begin{array}{l}\mathrm{N}^{\circ} \text { de planes de forma- } \\
\text { ción en centro }\end{array}$ & & & $\begin{array}{l}\text { Incremento del } N^{\circ} \text { de proyec- } \\
\text { tos desarrollados }\end{array}$ & & \\
\hline & & $\begin{array}{l}\text { \% de cumplimiento de } \\
\text { la programación }\end{array}$ & & & $\begin{array}{l}\mathrm{N}^{\circ} \text { actividades de cada } \\
\text { modalidad }\end{array}$ & & & $\begin{array}{l}\text { Grado de utilización de los } \\
\text { proyectos }\end{array}$ & & \\
\hline & & $\begin{array}{l}\text { Concretar el currículo } \\
\text { en el centro en base } \\
\text { al catálogo compe- } \\
\text { tencial }\end{array}$ & Vo \% & $\operatorname{Vr} \%$ & $\begin{array}{l}\text { Ofertar formación al } \\
\text { profesorado para el } \\
\text { desarrollo competen- } \\
\text { cial en el aula }\end{array}$ & Vo \% & $\operatorname{Vr} \%$ & $\begin{array}{l}\text { Crear plataformas de trabajo } \\
\text { colaborativo y de autoapren- } \\
\text { dizaje }\end{array}$ & Vo $\%$ & $\operatorname{Vr} \%$ \\
\hline & & $\begin{array}{l}\text { Grado de implemen- } \\
\text { tación }\end{array}$ & & & $\begin{array}{l}\text { Incremento del } \mathrm{N}^{\circ} \text { de } \\
\text { acciones formativas }\end{array}$ & & & $\mathrm{N}^{\circ}$ de espacios abiertos & & \\
\hline & & Grado de utilización & & & $\begin{array}{l}\mathrm{N}^{\circ} \text { actividades informa- } \\
\text { ción y difusión } \\
\mathrm{N}^{\circ} \text { de actividades on } \\
\text { line }\end{array}$ & & & $\mathrm{N}^{\circ}$ de usuarios registrados & & \\
\hline
\end{tabular}


TABLA IV.C CUADRO DE MANDO ESTRATÉGICO DEL SISTEMA EDUCATIVO EN BASE A LA LÍNEA ESTRATÉGICA DE LAS COMPETENCIAS. ESTRATEGIAS. POLÍTICAS PÚBLICAS.

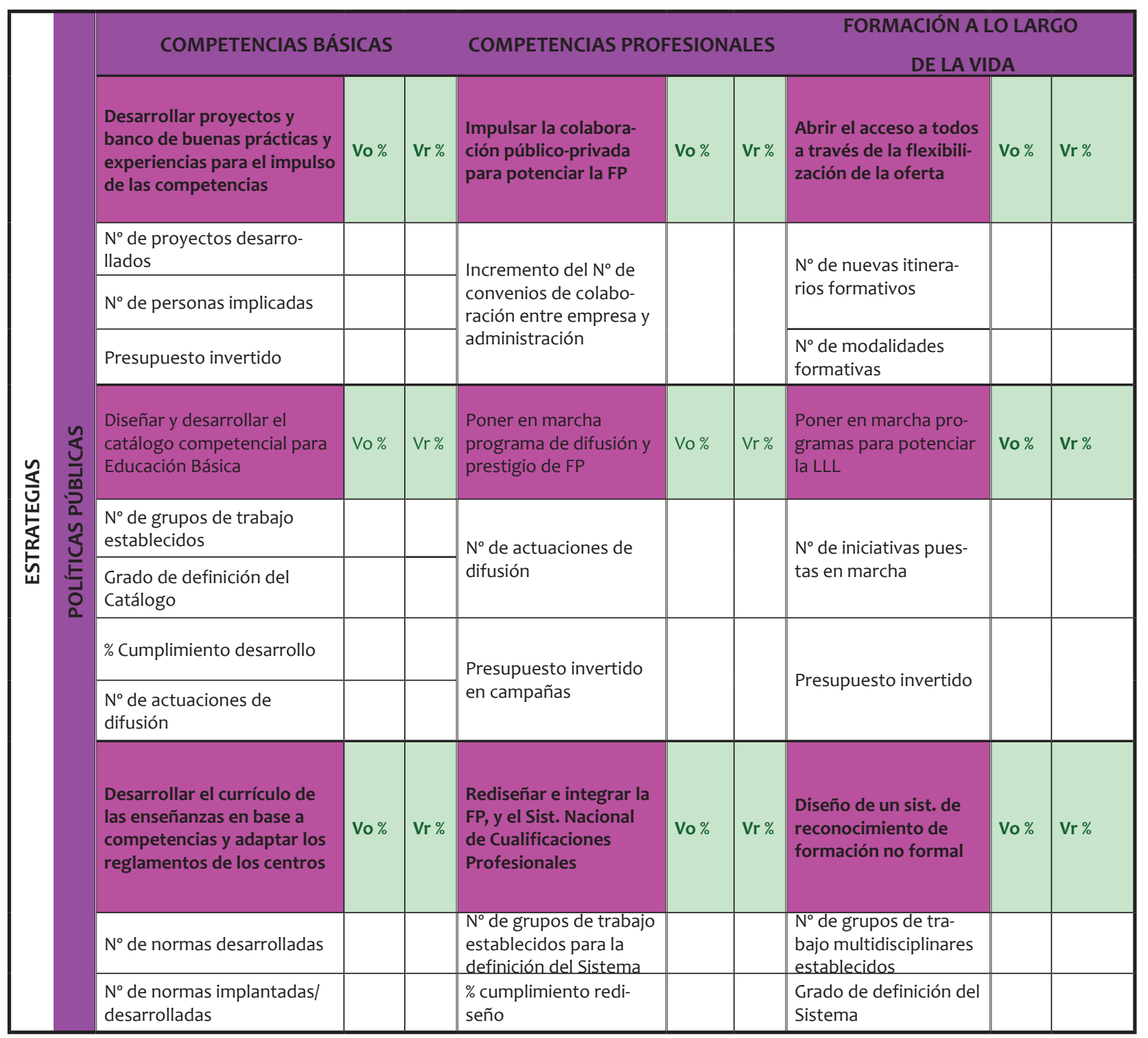

\section{Matriz de Alineación Estratégica (MAE) y Cuadro de Mando Integral (CMI)}

Ya hemos indicado antes que en este artículo nos centraríamos solamente en el mapa y el cuadro de mando estratégico, pero como colofón diremos que el siguiente paso sería el desarrollo de la MAE, que nos va a ayudar a alinear la estrategia de la organización recogida en el Mapa Estratégico con el despliegue a nivel operativo, siendo la principal entrada (input) a la hora de diseñar el mapa operativo y definiendo las actuaciones en los diferentes niveles de gestión: estratégico, táctico y operativo. La salida (output) o resultado de la matriz será la base para el desarrollo del Cuadro de Mando Integral, conformado por los indicadores de resultado asociados a los procesos, e igualmente que el CME agrupados por perspectivas y líneas estratégicas. El CMI sirve para monitorizar y retroalimentar el Modelo/Sistema periódicamente.

\section{CONCLUSIONES}

Todas las estadísticas, estudios e informes recientemente publicados (CIS,OCDE, INE), coinciden en los tres principales problemas de nuestro país: el empleo, la crisis económica y una escasa confianza en el sistema políticoadministrativo.

En todos ellos, se manifiesta que la educación y la formación debe ser uno de los impulsores que ayude a superar los dos primeros, y que la reforma de la administración, a partir de la trasparencia en la definición de las políticas públicas, y del impulso del buen gobierno de la administración pública, la solución al tercero. 
La implantación de la educación por competencias debe ser uno de los elementos en los que se sustente la mejora de los resultados del sistema educativo, y por ende los de la sociedad en su conjunto, así como la empleabilidad, la productividad y la competitividad de nuestras empresas. Y la utilización de métodos y herramientas como las mostradas en este artículo, han de permitir alinear el triángulo política pública-administración-ciudadano, aumentando la eficacia y eficiencia de las decisiones políticas y de la gestión pública, atendiendo las necesidades individuales y colectivas y consiguiendo así el doble objetivo de apoyar a la solución de los problemas a través de la generación de valor público.

El objetivo final de Modelo de Alineación Estratégica es hacer operativa la estrategia en todos los ámbitos de la implantación de las competencias, estableciendo las conexiones entre los distintos agentes y elementos del sistema educativo, de forma que se compartan los mismos objetivos estratégicos y que cada uno de estos elementos o agentes aporten valor desde sus ámbitos de influencia para la consecución de estos. Además permite la monitorización desde el diseño hasta la implantación, para disminuir los desajustes entre lo planificado y lo realizado, optimizando el proceso en todas sus fases, y dando respuesta de esta forma a las necesidades y expectativas de los ciudadanos y de la sociedad.

\section{BIBLIOGRAFÍA}

ARENILLA, M.

- (2011) Administración Pública entre dos siglos. (Homenaje a Mariano Baena del Alcazar). Madrid: INAP.

- (2011) Crisis y reforma de la Administración pública. Oleiros: Netbiblo.

ARIEU, Agustín (2007). Consistencia estratégica vs. acciones inconexas. España. MateriaBIZ.

BAENA DEL ALCAZAr, M.ARIANO (1995) “Reformando a Leviathan: La administración pública”. Revista Política y Sociedad.

BARDACH, EUGENE(1998): ), Los ocho pasos para el análisis de Políticas Públicas. Mexico: CIDE

BYRNE, JOHN A., GERDES,L.(2005). ), The Man Who Invented Management. New York, Bloomberg Businessweek Magazine

CHANDLER, ALFRED A. D., JR. (1980) Managerial Hierarchies. Harvard University Press.

CONSEJO ESCOLAR DEL ESTADO (2012), Informe 2012 sobre el Estado del Sistema Educativo. Curso 2010201. Madrid: Ministerio de Educación, Cultura y Deportes. Consejo Escolar del Estado. Secretaría General Técnica. Subdirección General de Documentación y Publicaciones. Edición: 2012. NIPO: 030-12-150-9.

FUNDACIÓN BERTELSMANN (2013), Juventud, empleo e iniciativa emprendedora en España. Madrid: FUNDACIÓN BERTELSMANN. Depósito Legal: B-10597-20133.

GALVANY, Albert A. (2012) Suntzui. El arte de la guerra. Traducción directa del chino antiguo (8a edición). Madrid: Trotta.

INSTITUTO NACIONAL DE EVALUACIÓN EDUCATIVA (2012), Sistema estatal de indicadores de la educación. EDICIÓN 2012. Madrid: Ministerio de Educación, Cultura y Deportes. Secretaría de Estado de Educación, Formación Profesional y Universidades. Instituto Nacional de Evaluación Educativa. Edita:@ Secretaría General Técnica. Subdirección General de Documentación y Publicaciones. Edición: 2012. NIPO: 030-12-419-5.

KAPLAN, R.S.Kaplan y Norton, D.P. Norton

- (2001), Como utilizar el Cuadro de Mando Integral ( $2^{\mathrm{a}}$ Ed). Barcelona: Gestión 2000.

- (2001), El Cuadro de Mando Integral (3 $3^{\mathrm{a}}$ Ed). Barcelona: Gestión 2000.

- (2006), Alignment. Barcelona: Gestión 2000.

- (2007). Mapas Estratégicos. Barcelona: Gestión 2000.

LÓPEZ CORREA, A. (2013), Henry Mintzberg: “El management es, de cierta manera, la esencia de la vida misma”. España. Managers Magazine.

MARTíN PÉREZ, S. y Martín Seco, P. (2014), La excelencia operativa en la Administración Pública Creando Valor Público: Guía para la implantación de la gestión basada en procesosen la Administración Pública. Madrid: INAP.

McCLELLAND, David D. C. (1971) Testing for Competence rather than for Intelligence, Educational Testing Service, Princeton, New Jersey, January 4.

GESTIÓN Y ANÁLISIS DE POLÍTICAS PÚBLICAS, Nueva Época, no 11 enero-junio 2014 ISSN: 1989-8991 - DOI: 10.24965/gapp.v0i11.10178 
MOORE, Mark H. (1998), Gestión estratégica y creación de valor en el sector público. Barcelona:Paidós

OECD (2012), Better Skills, Better Jobs, Better Lives: A Strategic Approach to Skills Policies, OECD Publishing. http://dx.doi.org/10.1787/9789264177338-en.

PANORAMA DE LA EDUCACIÓN INDICADORES DE LA OCDE 2012. INFORME ESPAÑOL. Ministerio de Educación, Cultura y Deportes. Secretaría de Estado de Educación, Formación Profesional y Universidades. Dirección General de Evaluación y Cooperación Territorial. Instituto Nacional de Evaluación Educativa. Madrid 2012.

PORTER, M. E. (2008). The Five Competitive Forces That Shape Strategy. Harvard Business Review.

RONDA PUPO, GUILLERMO A. Y MARCANÉ LASERRA, JOSÉ ÁNGEL, De la estrategia a la dirección estratégica. Un acercamiento a la integración de los niveles estratégico, táctico y operativo. Revista Ciencias de la Información abril 2004

RUIZ LÓPEZ, JOAQUÍN S. (2012): “Calidad en la Gestión Pública: del azar a la necesidad”. Revista CLAD Reforma y Democracia. Caracas. Venezuela.

SALGANIK, L. H, RYCHEN D. S., MOSER U., KONSTANT J. (1999), Projects on Competencies in the OECD Context: Analysis of Theoretical and Conceptual Foundations, SFSO, OECD, ESSI, Neuchâtel.

SUBIRATS HUMET, J., Varone, F., Larrue, C. (2008), Análisis y gestión de políticas públicas. Barcelona: Ariel.

TAYLOR, F.W. (1912) Scientific Management; Shop Management; Taylor's Testimony before the Special House Committee, Connecticut, Green wood Press.

VON NEWMAN, J. And Morgerstern, O (1944) Theory of games and economic behavior. Princenton Universtiy Press. Princenton, NJ. EEUU. 